\title{
More Grandma Identities
}

\author{
Sumit Kumar Jha \\ IIIT-Hyderabad, India \\ Email: kumarjha.sumit@research.iiit.ac.in
}

November 22, 2019

In the article [1], the author proves the following trigonometric identity (calling it the "grandma identity") valid for integer $m \geq 1$

$$
\prod_{k=1}^{m} 2 \cos \frac{k \pi}{2 m+1}=1
$$

In the article [2], the author proves following similar identities using (Girard) Newtons relations for symmetric polynomials, and the De Moivres formula. We prove all of these as consequences of a general proposition.

$$
\begin{gathered}
\prod_{k=1}^{2 m-1} \sin \frac{k \pi}{4 m}=\prod_{k=1}^{2 m-1} \cos \frac{k \pi}{4 m}=\sqrt{\frac{m}{2^{4 m-3}}} . \\
\prod_{k=1}^{m} \sin \frac{(2 k-1) \pi}{4 m}=\prod_{k=1}^{m} \cos \frac{(2 k-1) \pi}{4 m}=\frac{1}{2^{\frac{2 m-1}{2}}} . \\
\prod_{k=1}^{m} \sin \frac{k \pi}{2 m+1}=\prod_{k=1}^{m} \cos \frac{(2 k-1) \pi}{4 m+2}=\frac{\sqrt{2 m+1}}{2^{m}} . \\
\prod_{k=1}^{m} \cos \frac{k \pi}{2 m+1}=\prod_{k=1}^{m} \sin \frac{(2 k-1) \pi}{4 m+2}=\frac{1}{2^{m}} .
\end{gathered}
$$

The general proposition of which the above identities are consequences is

Proposition 1. For all complex numbers $z$ and all integers $n$, we have

$$
\sin n z=2^{n-1} \sin z \sin \left(z+\frac{\pi}{n}\right) \sin \left(z+\frac{2 \pi}{n}\right) \cdots \sin \left(z+\frac{(n-1) \pi}{n}\right) .
$$

Proof. We can write

$$
\begin{array}{r}
\sin n z=\frac{e^{n i z}-e^{-n i z}}{2 i}=\frac{e^{-n i z}\left(e^{2 n i z}-1\right)}{2 i} \\
=\frac{e^{-n i z}\left(e^{2 i z}-1\right)\left(e^{2 i z}-e^{\frac{-2 i \pi}{n}}\right) \cdots\left(e^{2 i z}-e^{\frac{-2(n-1) i \pi}{n}}\right)}{2 i} \\
=k \cdot \sin z \sin \left(z+\frac{\pi}{n}\right) \sin \left(z+\frac{2 \pi}{n}\right) \cdots \sin \left(z+\frac{(n-1) \pi}{n}\right)
\end{array}
$$

where

$$
k=(2 i)^{n-1} e^{\frac{-i \pi}{n}(1+2 \cdots+(n-1))}=(2 i)^{n-1} e^{\frac{-i \pi(n-1)}{2}}=2^{n-1} .
$$

1 PROOF OF IDENTITY (I)

To proceed with the proof of identity (1), we plug $n=4 m$ in (5) and let $z \rightarrow 0$ on both sides to get

$$
\prod_{k=1}^{4 m-1} \sin \frac{k \pi}{4 m}=\frac{1}{2^{4 m-1}} \lim _{z \rightarrow 0} \frac{\sin 4 m z}{\sin z}=\frac{m}{2^{4 m-3}} .
$$


But

$$
\prod_{k=1}^{4 m-1} \sin \frac{k \pi}{4 m}=\prod_{k=1}^{2 m-1} \sin \frac{k \pi}{4 m} \prod_{k=2 m+1}^{4 m-1} \sin \left(\pi-\frac{k \pi}{4 m}\right)=\prod_{k=1}^{2 m-1} \sin ^{2} \frac{k \pi}{4 m}
$$

which proves first part of (1). The second part of (1) follows from the fact

$$
\cos \frac{k \pi}{4 m}=\sin \left(\frac{\pi}{2}-\frac{k \pi}{4 m}\right)=\sin \frac{(2 m-k) \pi}{4 m} .
$$

2 PROOF OF IDENTITY (2)

To prove identity (2), we first let $z \rightarrow-\pi / 2 n$ on both sides of (5) to get

$$
1=2^{n-1} \sin \frac{\pi}{2 n} \prod_{k=1}^{n-1} \sin \left(-\frac{\pi}{2 n}+\frac{k \pi}{n}\right)=2^{n-1} \sin \frac{\pi}{2 n} \prod_{k=1}^{n-1} \sin \frac{(2 k-1) \pi}{2 n}
$$

Plugging $n=2 m$ in the above gives us

$$
\frac{1}{2^{2 m-1}}=\sin \frac{\pi}{4 m} \prod_{k=1}^{2 m-1} \sin \frac{(2 k-1) \pi}{4 m}
$$

But

$$
\begin{array}{r}
\sin \frac{\pi}{4 m} \prod_{k=1}^{2 m-1} \sin \frac{(2 k-1) \pi}{4 m}=\sin \frac{\pi}{4 m} \prod_{k=1}^{m} \sin \frac{(2 k-1) \pi}{4 m} \prod_{k=m+1}^{2 m-1} \sin \left(\pi-\frac{(2 k-1) \pi}{4 m}\right) \\
=\prod_{k=1}^{m} \sin ^{2} \frac{(2 k-1) \pi}{4 m} .
\end{array}
$$

This proves the first part of (2). The second part of (2) follows from fact that

$$
\prod_{k=1}^{m} \cos \frac{(2 k-1) \pi}{4 m}=\prod_{k=1}^{m} \cos \frac{(2 m-2 k+1) \pi}{4 m}=\prod_{k=1}^{m} \cos \left(\frac{\pi}{2}-\frac{(2 k-1) \pi}{4 m}\right) .
$$

3 PROOF OF IDENTITY (3)

To prove identity (3), we plug $n=2 m+1$ and let $z \rightarrow 0$ on both sides of (5) to get

$$
\prod_{k=1}^{2 m} \sin \frac{k \pi}{2 m+1}=\frac{1}{2^{2 m}} \lim _{z \rightarrow 0} \frac{\sin (2 m+1) z}{\sin z}=\frac{2 m+1}{2^{2 m}} .
$$

But

$$
\prod_{k=1}^{2 m} \sin \frac{k \pi}{2 m+1}=\prod_{k=1}^{m} \sin \frac{k \pi}{2 m+1} \prod_{k=m+1}^{2 m} \sin \left(\pi-\frac{k \pi}{2 m+1}\right)=\prod_{k=1}^{m} \sin ^{2} \frac{k \pi}{2 m+1} .
$$

This proves the first part of the identity (3). The second part of (3) follows from the fact that

$$
\begin{array}{r}
\prod_{k=1}^{m} \cos \frac{(2 k-1) \pi}{4 m+2}=\prod_{k=1}^{m} \sin \left(\frac{\pi}{2}-\frac{(2 k-1) \pi}{4 m+2}\right) \\
=\prod_{k=1}^{m} \sin \frac{(m-k+1) \pi}{2 m+1}=\prod_{k=1}^{m} \sin \frac{k \pi}{2 m+1} .
\end{array}
$$

\section{PROOF OF IDENTITY (4)}

To prove identity (4), we plug $n=2 m+1$ in (6) to get

$$
\frac{1}{2^{2 m}}=\sin \frac{\pi}{4 m+2} \prod_{k=1}^{2 m} \sin \frac{(2 k-1) \pi}{4 m+2} .
$$




$$
\begin{array}{r}
\sin \frac{\pi}{4 m+2} \prod_{k=1}^{2 m} \sin \frac{(2 k-1) \pi}{4 m+2}=\sin \frac{\pi}{4 m+2} \prod_{k=1}^{m} \sin \frac{(2 k-1) \pi}{4 m+2} \prod_{k=m+1}^{2 m} \sin \left(\pi-\frac{(2 k-1) \pi}{4 m+2}\right) \\
=\prod_{k=1}^{m} \sin ^{2} \frac{(2 k-1) \pi}{4 m+2} .
\end{array}
$$

This proves the first part of (4). The second part of (4) follows from the fact that

$$
\prod_{k=1}^{m} \sin \frac{(2 k-1) \pi}{4 m+2}=\prod_{k=1}^{m} \cos \left(\frac{\pi}{2}-\frac{(2 k-1) \pi}{4 m+2}\right)=\prod_{k=1}^{m} \cos \frac{k \pi}{2 m+1} .
$$

REFERENCES

1. Humble, Steve. "88.62 Grandma's identity." The Mathematical Gazette 88 , no. 513 (2004): 524-525.

2. Amrik Singh Nimbran. (2017). An Infinite Product for the Square Root of an Integer. The American Mathematical Monthly, 124(7), 647-650. doi:10.4169/amer.math.monthly.124.7.647 